\title{
Oclusopatias e necessidade de tratamento ortodôntico em adolescentes de 12 anos de
}

\section{idade}

\author{
Malocclusion and the need for orthodontic treatment in 12-year-old adolescents \\ Maloclusión y necesidad de tratamiento de ortodoncia en adolescentes de 12 años
}

\section{Resumo}

O tratamento das oclusopatias em um sistema de saúde pública requer o levantamento de dados sobre as necessidades ortodônticas de uma determinada população. Dessa forma, diferentes métodos de registro foram desenvolvidos para classificar os problemas de oclusão. O objetivo neste estudo foi investigar a prevalência das oclusopatias e a necessidade normativa de tratamento ortodôntico em jovens de 12 anos de idade. Trata-se de um estudo epidemiológico transversal realizado com 461 escolares da rede de ensino público. Para a avaliação foram realizados exames clínicos utilizando a Classificação de Angle e o Índice de Estética Dentária (DAI). Observou-se que 8,89\% apresentavam oclusão normal, 56,83\% oclusopatia em Classe I, 24,08\% em Classe II e 10.20\% em Classe III. Nenhum ou necessidade de tratamento ortodôntico leve foi observado em 47,51\%; o tratamento eletivo esteve presente em 20,61\%, altamente desejável em 13,45\% e obrigatório em 18,44\% dos jovens. Dentre os resultados dos exames clínicos para diagnóstico de oclusopatias utilizando a Classificação de Angle foi observado associação com o Índice de Estética Dentária (DAI) (p <0,0001). Dos componentes do DAI, as principais anomalias dentofaciais observadas foram o apinhamento $(63,56 \%)$, desalinhamento mandibular anterior $(62,26 \%)$ e overjet maxilar $(45,99 \%)$. A prevalência das oclusopatias e da necessidade normativa de tratamento ortodôntico foi elevada, sendo que aproximadamente $1 / 5 \mathrm{com}$ necessidade de tratamento obrigatório. Os índices foram eficazes para a identificação das oclusopatias, entretanto por avaliarem aspectos clínicos distintos, poderiam ser utilizados concomitantemente para aprimorar o diagnóstico das oclusopatias.

Palavras-chave: Má oclusão; Epidemiologia; Saúde pública.

\begin{abstract}
The treatment of malocclusions in a public health system requires the collection of data on the orthodontic needs of a given population. Thus, different recording methods have been developed to classify occlusion problems. The objective in this study was to investigate the prevalence of malocclusions and the normative need for orthodontic treatment in 12-year-olds. This is a cross-sectional epidemiological study carried out with 461 students from public schools. For the evaluation, clinical examinations were performed using the Angle's Classification and the Dental Aesthetics Index (DAI). It was observed that $8.89 \%$ had normal occlusion, $56.83 \%$ Class I, 24.08\% Class II and $10.20 \%$ Class III. None or need for mild orthodontic treatment was observed in $47.51 \%$; elective treatment was
\end{abstract}


present in $20.61 \%$, highly desirable in $13.45 \%$ and mandatory in $18.44 \%$ of young people. Among the results of clinical examinations for the diagnosis of malocclusions using the Angle's Classification, an association with the Dental Aesthetic Index (DAI) was observed ( $p<0.0001)$. Of the components of the DAI, the main dentofacial anomalies observed were crowding (63.56\%), anterior mandibular misalignment $(62.26 \%)$ and maxillary overjet $(45.99 \%)$. The prevalence of malocclusions and the normative need for orthodontic treatment was high, with approximately $1 / 5$ of the sample needing mandatory treatment. The indices were effective for the identification of malocclusions, however, by evaluating different clinical aspects, they could be used concomitantly to improve the diagnosis of malocclusions.

Keywords: Malocclusion; Epidemiology; Public health.

\section{Resumen}

El tratamiento de las maloclusiones en un sistema de salud pública requiere la recolección de datos sobre las necesidades de ortodoncia de una población determinada. Por tanto, se han desarrollado diferentes métodos de registro para clasificar los problemas de oclusión. El objetivo de este estudio fue investigar la prevalencia de maloclusiones y la necesidad normativa de tratamiento de ortodoncia en niños de 12 años. Se trata de un estudio epidemiológico transversal realizado con 461 estudiantes de escuelas públicas. Para la evaluación, se realizaron exámenes clínicos utilizando la Clasificación de Angle y el Índice de Estética Dental (DAI). Se observó que 8.89\% tuvo oclusión normal, $56.83 \%$ Clase I, $24.08 \%$ Clase II y $10.20 \%$ Clase III. No se observó ninguna o necesidad de tratamiento de ortodoncia leve en el 47,51\%; El tratamiento electivo estuvo presente en el 20,61\%, muy deseable en el $13,45 \%$ y obligatorio en el 18,44\% de los jóvenes. Entre los resultados de los exámenes clínicos para el diagnóstico de maloclusiones utilizando la Clasificación de Angle, se observó una asociación con el Índice de Estética Dental (DAI) ( $\mathrm{p}<0,0001)$. De los componentes del DAI, las principales anomalías dentofaciales observadas fueron apiñamiento $(63,56 \%)$, desalineación mandibular anterior $(62,26 \%)$ y resalte maxilar $(45,99 \%)$. La prevalencia de maloclusiones y la necesidad normativa de tratamiento de ortodoncia fue alta, con aproximadamente 1/5 requiriendo tratamiento obligatorio. Los índices resultaron efectivos para la identificación de maloclusiones, sin embargo, al evaluar diferentes aspectos clínicos, se pudieron utilizar de forma concomitante para mejorar el diagnóstico de maloclusiones.

Palabras clave: Maloclusión; Epidemiología; Salud pública.

\section{Introdução}

As oclusopatias representam um importante problema de saúde pública por apresentarem alta prevalência, possibilidade de prevenção e tratamento e promoverem prejuízos significantes na qualidade de vida dos indivíduos acometidos (World Health Organization, 2003; Chaves, 1986). Assim, torna-se fundamental a realização de estudos que investiguem a distribuição, a severidade e as consequências das oclusopatias em diferentes populações, visando contribuir para o desenvolvimento de estratégias e políticas públicas que minimizem a instalação e o agravamento das oclusopatias (World Health Organization, 2003; Chaves, 1986). Um estudo de base populacional brasileiro realizado em 2010 mostrou que 38,8\% dos adolescentes de 12 anos apresentavam alterações na oclusão (Brasil, 2009). À nível global, Alhammadi MS et al. (2018) utilizou a Classificação de Angle e observou que as médias para Classe I, II e III foram 74,7\%, 19,56\% e 5,93\%, respectivamente, na dentição permanente e $73 \%, 23 \%$ e $4 \%$ na dentição mista.

Para o tratamento adequado das oclusopatias em um sistema de saúde pública, é importante o levantamento de dados sobre as necessidades ortodônticas de uma determinada população (Garbin et al., 2010). Dessa forma, diferentes métodos de registro foram desenvolvidos para classificar os problemas de oclusão (Pinto et al., 2008; Ashari et al., 2016). A Classificação de Angle (Angle, 1899) pode ser considerada pioneira na evolução da área ortodôntica; tanto por explanar a categorização das oclusopatias quanto por elucidar, de forma simples, o conceito de oclusão normal (Hassan et al., 2007). Esse método, apesar de ser um indicador qualitativo e apresentar limitações para estudos epidemiológicos, tem se mantido, amplamente, aceito e preconizado nas instituições de ensino em odontologia e, possivelmente, pode ser considerado o instrumento mais utilizado para o registro das oclusopatias na prática e nos estudos em ortodontia (Pinto et al., 2008).

A Organização Mundial da Saúde (OMS), a partir de 1997, proporcionou mudanças na mensuração das oclusopatias e recomendou o uso do Índice de Estética Dentária (DAI). É considerado um índice transcultural que tem sido aplicado em diversos grupos étnicos (Ashari et al., 2016; Vedovello et al. 2019), permite o registro de natureza quantitativa e a coleta de dados em caráter epidemiológico (Garbin et al., 2010). Este índice pode ser utilizado para estimar e priorizar a necessidade de 
tratamento ortodôntico da população, principalmente onde há limitação de recursos nos serviços de saúde (Tolessa et al., 2020).

Embora o crescimento no número de estudos direcionados às oclusopatias em diferentes populações esteja presente na literatura, é uma temática em discussão por muitos anos, devido à dificuldade em obter a padronização internacional ideal para o registro dos problemas de oclusão. Desta forma, o objetivo do estudo foi investigar a prevalência das oclusopatias e a necessidade normativa de tratamento ortodôntico em jovens de 12 anos de idade utilizando a Classificação de Angle e o DAI.

\section{Metodologia}

\section{Critérios Éticos}

O estudo foi submetido e aprovado pelo Comitê de Ética em Pesquisa e conduzido de acordo com as normas constantes na Declaração de Helsinque e nas Resoluções do Conselho Nacional de Saúde nº 466, de 12 de dezembro de 2012 com protocolo número 11036219.9.0000.5420. Um Termo de Consentimento e um Termo de Assentimento foram utilizados para os participantes da pesquisa.

\section{Composição da amostra}

Consistiu-se em um estudo epidemiológico transversal, composto por jovens, em ambos os sexos, com 12 anos de idade, regularmente matriculados em escolas da Rede de Ensino Público de um município de médio porte do Estado de São Paulo.

O tamanho da amostra foi calculado considerando que o número de indivíduos com necessidade de tratamento ortodôntico pertencentes à população do estudo não era conhecido e adotando uma margem de erro de 5\% e um nível de confiança de $95 \%$, a amostra mínima determinada foi 344 de participantes.

Todos os escolares regularmente matriculados com 12 anos completos e/ou que completariam esta idade no ano da pesquisa foram convidados a participar no período de agosto a dezembro de 2019, compondo total de 461 estudantes examinados.

Foram excluídos do estudo aqueles que negaram a participação do exame, os pais e/ou responsáveis legais que não assinaram o Termo de Consentimento Livre e Esclarecido (TCLE) e os que se ausentaram após a terceira tentativa de coleta de dados.

\section{Instrumentos da pesquisa}

Para a avaliação das oclusopatias e da necessidade de tratamento ortodôntico foram utilizados instrumentos baseados nos critérios da Classificação de Angle (Angle, 1899) e DAI (Brasil, 2009).

Um questionário sociodemográfico também foi utilizado para a caracterização da população, indicando o grupo étnico (branco, não-branco, não declarou), a renda familiar (em reais) (até 500, de 501,00 a 1500,00, de 1501,00 a 2500,00, mais de 2500, não declarou) e o nível de escolaridade dos pais e/ou responsáveis legais (analfabeto, fundamental incompleto/completo, médio incompleto/completo, superior incompleto/completo, não declarou), baseadas nos aspectos metodológicos do Projeto SBBrasil 2010 (Brasil, 2009).

\section{Coleta de dados}

Foi realizada a conscientização com os alunos de 12 anos de idade e professores, em sala de aula, sobre os problemas de saúde bucal, as oclusopatias e a necessidade de tratamento ortodôntico. Por meio da aplicação de um questionário estruturado foram coletadas informações sobre a condição sociodemográfica dos adolescentes e por meio da realização de 
exames clínicos bucais foram obtidos dados sobre a presença, severidade e necessidade de tratamento de oclusopatias.

\section{Exames clínicos}

Foi realizada uma calibração intraexaminador prévia, objetivando-se encontrar possíveis dificuldades de manuseio dos métodos de registro utilizados no estudo. O coeficiente Kappa intraexaminador obtido no estudo piloto foi de 0,90.

Os exames clínicos para avaliação das oclusopatias foram realizados por uma única equipe composta por um examinador e um anotador, previamente treinados, no ambiente do pátio das escolas, com adequadas condições de ventilação e iluminação natural, utilizando sonda periodontal da OMS e espelho bucal plano. Os alunos foram organizados de modo que os exames fossem realizados individualmente, evitando aglomerações e preservando o sigilo das informações.

\section{Análise estatística}

Os dados foram analisados empregando-se técnicas de estatística descritiva e as distribuições absolutas e percentuais das variáveis categóricas foram apresentadas por meio de tabelas. A verificação da associação entre os resultados dos exames clínicos para diagnóstico de oclusopatias utilizando a Classificação de Angle e o DAI foi realizada por meio do teste G com correção de Williams, adotando-se um nível de significância de 5\%. O processamento e a análise dos dados foram realizados com auxílio do Programa Epi-InfoTM versão 7.2. (Center for Disease Control and Prevention).

\section{Resultados}

Do total da amostra obtida ( $\mathrm{n}=461)$, participaram do estudo $226(49,02 \%)$ estudantes do sexo masculino e 235 (50,98\%) feminino. Na declaração da cor da pele, $43.60 \%$ eram brancos, 29,07\% dos responsáveis legais relataram não ter completado o ensino fundamental e a renda familiar predominante foi equivalente à até $\mathrm{R} \$ 1500,00$, conforme os dados expressados na Tabela 1. 
Tabela 1: Distribuição das frequências absoluta (n) e percentual (\%) da população do estudo, segundo o sexo, a cor, escolaridade e renda familiar, no município de Araçatuba, São Paulo, 2019.

\begin{tabular}{|c|c|c|}
\hline \multicolumn{3}{|l|}{ Dados sociodemográficos } \\
\hline Variáveis & $\mathrm{n}$ & $\%$ \\
\hline \multicolumn{3}{|l|}{ Sexo } \\
\hline Masculino & 226 & 49.02 \\
\hline Feminino & 235 & 50.98 \\
\hline \multicolumn{3}{|l|}{ Cor } \\
\hline Branco & 201 & 43.60 \\
\hline Não-Branco & 200 & 43.38 \\
\hline Não declarou & 60 & 13.02 \\
\hline \multicolumn{3}{|l|}{ Escolaridade } \\
\hline Analfabeto & 1 & 0.22 \\
\hline Fundamental incompleto & 134 & 29.07 \\
\hline Fundamental completo & 54 & 11.71 \\
\hline Médio incompleto & 80 & 17.35 \\
\hline Médio completo & 69 & 14.97 \\
\hline Superior incompleto & 9 & 1.95 \\
\hline Superior completo & 17 & 3.69 \\
\hline Não declarou & 99 & 21.48 \\
\hline \multicolumn{3}{|l|}{ Renda familiar (R\$) } \\
\hline Até 500,00 & 33 & 7.16 \\
\hline De 501,00 a 1500,00 & 190 & 41.21 \\
\hline De 1501,00 a 2500,00 & 55 & 11.93 \\
\hline Mais que 2500,00 & 29 & 6.29 \\
\hline Não declarou & 154 & 33.41 \\
\hline Total & 461 & 100.00 \\
\hline
\end{tabular}

Considerando a Classificação de Angle, menos de $10 \%$ dos jovens examinados apresentavam oclusão normal e a maioria foram registrados com oclusopatia, conforme demonstrado na Tabela 2. Dentre os jovens com oclusopatia, mais da metade apresentou problemas de oclusão em Classe I e aproximadamente 10\% em Classe III. As crianças com oclusopatias em Classe II, $61(54,95 \%)$ foram categorizados como divisão 1 e 50 (49,05\%) como divisão 2. 
Tabela 2: Distribuição das frequências absoluta (n) e percentual (\%) das oclusopatias de jovens de 12 anos de idade, segundo a Classificação de Angle, no município de Araçatuba, São Paulo, 2019.

\begin{tabular}{lrr}
\hline Oclusopatia & $\mathrm{n}$ & $\%$ \\
\hline Oclusão normal & 21 & 8.89 \\
Classe I & 282 & 56.83 \\
Classe II & 111 & 24.08 \\
Classe III & 47 & 10.20 \\
Total & 461 & 100.00 \\
\hline \multicolumn{2}{c}{}
\end{tabular}

Em relação ao DAI, considerando a necessidade de tratamento ortodôntico eletivo à obrigatório, a prevalência foi de $52,5 \%$, de acordo com a Tabela 3.

Tabela 3: Distribuição das frequências absoluta (n) e percentual (\%) da necessidade de tratamento ortodôntico de jovens de 12 anos de idade, segundo o Índice de Estética Dentária (DAI), no município de Araçatuba, São Paulo, 2019.

\begin{tabular}{ccccc}
\hline DAI & Severidade de oclusopatia & $\begin{array}{c}\text { Necessidade tratamento } \\
\text { ortodôntico }\end{array}$ & $\mathrm{n}$ & $\%$ \\
\hline$\leq 25$ & Sem anormalidade ou oclusopatia & Nenhum ou tratamento leve & 219 & 47.51 \\
26 a 30 & Má oclusão definida & Eletivo & 95 & 20.61 \\
31 a 35 & Má oclusão severa & Altamente desejável & 62 & 13.45 \\
$>35$ & Má oclusão muito severa ou & Obrigatório & 85 & 18.44 \\
Total & incapacitante & & 461 & 100.00 \\
\hline
\end{tabular}

Fonte: Autores.

Dentre os componentes do DAI, as principais anomalias dentofaciais ocorreram na dimensão espaço com destaque para o apinhamento $(63,56 \%)$ e o desalinhamento mandibular anterior $(62,26 \%)$ e na dimensão oclusão, o overjet maxilar $(45,99 \%)$, conforme a Tabela 4. 
Tabela 4: Distribuição das frequências absoluta (n) e percentual (\%) dos componentes do DAI (dentição, espaço e oclusão) em jovens de 12 anos de idade, no município de Araçatuba, São Paulo, 2019.

\begin{tabular}{|c|c|c|c|c|}
\hline \multirow{2}{*}{ Componentes do DAI } & \multicolumn{2}{|c|}{ Presente } & \multicolumn{2}{|c|}{ Ausente } \\
\hline & $\mathrm{n}$ & $\%$ & $\mathrm{n}$ & $\%$ \\
\hline \multicolumn{5}{|l|}{ Dentição } \\
\hline Perda dentária anterior & 14 & 3.04 & 447 & 96.96 \\
\hline \multicolumn{5}{|l|}{ Espaço } \\
\hline Apinhamento & 293 & 63.56 & 168 & 36.44 \\
\hline Espaçamento & 146 & 31.67 & 315 & 68.33 \\
\hline Diastema & 146 & 31.67 & 315 & 68.33 \\
\hline Desalinhamento maxilar anterior & 188 & 40.78 & 273 & 59.22 \\
\hline Desalinhamento mandibular anterior & 287 & 62.26 & 174 & 37.74 \\
\hline \multicolumn{5}{|l|}{ Oclusão } \\
\hline Overjet maxilar & 212 & 45.99 & 249 & 54.01 \\
\hline Overjet mandibular & 21 & 4.56 & 440 & 95.44 \\
\hline Mordida aberta anterior & 18 & 3.9 & 443 & 96.1 \\
\hline
\end{tabular}

Fonte: Autores.

Ainda na dimensão oclusão dos componentes do DAI, observou-se que 34,27\% apresentavam alteração na relação molar antero-posterior, como visto na Tabela 5.

Tabela 5: Distribuição das frequências absoluta (n) e percentual (\%) da relação molar antero-posterior de jovens de 12 anos de idade, segundo o Índice de Estética Dentária (DAI), no município de Araçatuba, 2019.

\begin{tabular}{lcr}
\hline \multicolumn{1}{c}{ Relação molar antero-posterior } & $\mathrm{n}$ & $\%$ \\
\hline Normal & 303 & 65.73 \\
Meia cúspide & 101 & 21.91 \\
Uma cúspide & 57 & 12.36 \\
Total & 461 & 100.00 \\
\hline
\end{tabular}

Fonte: Autores.

Foi possível verificar associação $(\mathrm{p}<0,0001)$ entre os resultados dos exames clínicos para diagnóstico de oclusopatias utilizando a Classificação de Angle e o DAI. Mais da metade do total da amostra apresentavam o score DAI $\leq 25$. Neste score foram registradas todas as crianças classificadas com oclusão normal, 58,45\% em Classe I, 20,55\% em Classe II e 11,42\% Classe em III, segundo a classificação de Angle. Uma parcela considerável apresentava Classe I Angle com presença de anomalias dentofaciais distribuídas nos diferentes níveis de necessidade de tratamento ortodôntico, de acordo com os escores do DAI, conforme a Tabela 6.

Tabela 6: Distribuição absoluta (n) e percentual (\%) do tipo de oclusão, segundo a comparação entre o Índice de Estética Dentária (DAI) e a Classificação de Angle dos jovens de 12 anos de idade no município de Araçatuba, São Paulo, 2019. (p $<0,0001)$ para associação entre os resultados da Classificação de Angle e do Índice de Estética Dentária (DAI). 


\begin{tabular}{|c|c|c|c|c|c|c|c|c|c|c|}
\hline \multicolumn{11}{|c|}{ Classificação de Angle } \\
\hline \multirow[t]{2}{*}{ DAI } & \multicolumn{2}{|c|}{ Oclusão Normal } & \multicolumn{2}{|c|}{ Classe I } & \multicolumn{2}{|c|}{ Classe II } & \multicolumn{2}{|c|}{ Classe III } & \multicolumn{2}{|c|}{ Total } \\
\hline & $\mathrm{n}$ & $\%$ & $\mathrm{n}$ & $\%$ & $\mathrm{n}$ & $\%$ & $\mathrm{n}$ & $\%$ & $\mathrm{n}$ & $\%$ \\
\hline$\leq 25$ & 21 & 9.59 & 128 & 58.45 & 45 & 20.55 & 25 & 11.42 & 219 & 100.00 \\
\hline 26 a 30 & 0 & 0.00 & 64 & 67.37 & 22 & 23.16 & 9 & 9.47 & 95 & 100.00 \\
\hline 31 a 35 & 0 & 0.00 & 41 & 66.13 & 13 & 20.97 & 8 & 12.90 & 62 & 100.00 \\
\hline$>35$ & 0 & 0.00 & 49 & 57.65 & 31 & 36.47 & 5 & 5.88 & 85 & 100.00 \\
\hline Total & 21 & & 282 & & 111 & & 47 & & 461 & \\
\hline
\end{tabular}

Fonte: Autores.

\section{Discussão}

O objetivo neste estudo foi investigar a necessidade de tratamento ortodôntico, em resposta à alta prevalência das oclusopatias. Segundo a Organização Mundial da Saúde (World Health Organization, 1987), esta condição é considerada um problema em saúde pública e ocupa a terceira posição em uma escala prioritária dos problemas bucais, com base em informações epidemiológicas nacionais e internacionais.

No território brasileiro, o primeiro levantamento epidemiológico de saúde bucal no âmbito nacional (Brasil, 2003) a avaliar as oclusopatias foi realizado entre os períodos 2002-2003 e apontou que 58,1\% dos jovens de 12 anos de idade apresentaram problemas de oclusão (Brasil, 2003). Mais adiante, os resultados da Pesquisa Nacional de Saúde Bucal, em 2010, mostraram que cerca de 38,9\% das crianças aos 12 anos de idade apresentaram oclusopatias (Brasil, 2009).

A partir destes dados, torna-se evidente que a avaliação periódica e regular da prevalência da oclusopatia e da necessidade de tratamento, principalmente, sob a perspectiva da saúde pública, são essenciais para o conhecimento da realidade epidemiológica da população, a colaboração na determinação da prioridade de tratamento nos serviços odontológicos e o planejamento dos recursos necessários para assistir a demanda existente (Esa et al., 2001; Gudipaneni et al.,2018).

Para a avaliação da prevalência da oclusopatia, o método de registro utilizado neste estudo foi a Classificação de Angle e os dados mostraram que as principais alterações foram em Classe I (56,83\%) e Classe II (24,08\%). Há evidências na literatura que observaram maior concentração de indivíduos com problemas de oclusão em Classe I, seguido das oclusopatias em Classe II e III (Alhammadi e al., 2018; Todor et al., 2019, Asiry et al., 2019, Lin et al., 2020). Uma atual revisão sistemática e meta-análise, de Lombardo et. al. (2020), quantificou, em escala global, a prevalência e o tipo de oclusopatias em crianças e adolescentes nas diferentes fases da dentição utilizando a Classificação de Angle; foram observadas médias de 55,5\% para Classe I, 24,7\% para Classe II e 10,7\% para Classe III, assemelhando-se aos resultados nesta investigação.

Para a avaliação da necessidade de tratamento ortodôntico foi utilizado o DAI. Fundamenta-se em uma combinação de medidas as quais, em conjunto, apresentam a finalidade de expressar o estado oclusal do indivíduo e, consequentemente, categorizá-lo em um nível de necessidade de tratamento ortodôntico, devido à composição do índice, que considera o comprometimento estético, além da oclusão (Brasil, 2012; Ashira et al., 2016). Estudos realizados no Brasil, Índia, África, China, entre outros mostraram elevada necessidade de tratamento ortodôntico aos 12 anos de idade (de Almeida et al., 2003; Herkrath et al.2019; Nayak et al.,2015; van Wyk et al., 2005; Baram et al., 2019). Estes resultados corroboraram com os resultados neste estudo, em que 52,5\% das crianças carecia de assistência ortodôntica, sendo 13,45\% altamente desejável e $18,44 \%$ obrigatória.

Em relação aos componentes do DAI, ao todo são 10 medidas avaliadas considerando três dimensões: dentição, espaço e oclusão (Ashira et al., 2016). Na dentição, quase a totalidade apresentavam a região anterior completa. Àqueles com 
registro de perda dentária anterior não possuíam o decíduo canino em posição no momento da coleta de dados (3,04\%). Savara \& Steen (1978) observaram que, no processo eruptivo, os caninos e os segundos molares permanentes eram os últimos elementos a se manifestarem na cavidade oral, completando a segunda dentição. Pressupõe-se que a cronologia de erupção dos dentes permanentes pode ter influenciado neste estudo.

Sobre a dimensão espaço, as condições que apresentaram as maiores frequências foram o apinhamento na região dos incisivos (63,56\%) e o desalinhamento mandibular anterior (40,78\%). Dentre as anomalias dentofaciais relacionadas aos problemas de espaço e ao impacto na estética dentária podem ser citados o apinhamento e o desalinhamento (Nunes Neto et al., 2014). Nunes Neto et al. (2014) e Ting et al. (2011) investigaram fatores associados aos problemas de espaço e ambos sugeriram que a genética poderia contribuir para a etiologia. Acredita-se que o padrão de crescimento facial seja um importante fator hereditário que favorece o desenvolvimento de oclusopatias (Moyers, 1988). Sugere-se que a predisposição genética pode ter influenciado nos resultados neste estudo, na medida em que o Brasil possui um grau considerável de miscigenação racial.

Além da genética, fatores ambientais podem colaborar para o desenvolvimento das oclusopatias. Segundo Doğramac1 et al. (2016), o comportamento de sucção não nutritiva pode ser considerado um fator de risco para problemas ortodônticos. Proffit et al. (2013) e Thomaz et al. (2013) observaram que estes hábitos, principalmente a sucção digital podem contribuir, especificamente, para o desenvolvimento de overjet maxilar. Sugere-se que estas evidências poderiam ter influenciado na maior frequência desta anomalia dentofacial neste estudo na dimensão oclusão dos componentes do DAI.

Com relação aos resultados dos exames clínicos utilizando a Classificação de Angle e o DAI para avaliação da prevalência de oclusopatias e a necessidade de tratamento ortodôntico, estudos (Garbin et al., 2010; Kaygisiz et al., 2016) observaram que houve associação entre os índices ( $\mathrm{p}<0,0001$ ), assim como, neste estudo. Foi possível verificar que do total da amostra $(n=461)$, mais da metade das crianças apresentavam o score $\mathrm{DAI} \leq 25$, ou seja, sem anormalidade ou oclusopatia leve e necessidade de nenhum ou tratamento leve. Neste score foram registradas todas as crianças classificadas com oclusão normal, 58,45\% em Classe I, 20,55\% em Classe II e 11,42\% Classe em III, segundo a Classificação de Angle. Sugere-se oclusopatia leve nas Classes I, II e III, uma vez que, ao observar a relação molar antero-posterior no DAI, a maioria apresentava posição molar normal $(65,73 \%)$ e o primeiro molar inferior deslocado meia cúspide para a mesial ou distal da relação de oclusão normal (21,91\%). Em um estudo de Silva et al. (2016) não houve associação entre os métodos e foi observado que a Classificação de Angle pode superestimar a necessidade de tratamento ortodôntico.

Dessa forma, apesar dos métodos de registro neste estudo terem expressado dados importantes sobre a associação entre a condição das oclusopatias e a necessidade de tratamento ortodôntico, os resultados devem ser comparados a outros estudos com cautela, devido às diferenças relacionadas à seleção dos participantes, estratégias diagnósticas, limitações dos instrumentos de avaliação, etnias e idade entre as amostras (Borzabadi-Farahani et al., 2011; Silva et al., 2016).

Quanto às limitações, ambos os métodos utilizados não mostraram sensibilidade à alguns problemas de oclusão, o que poderia restringir os resultados em estudos epidemiológicos. Apesar das vantagens que o DAI apresenta, incluindo alta confiabilidade e validade, exclui molares ausentes, dentes impactados, mordida cruzada, mordida profunda e discrepâncias na linha média do cálculo dos escores (Kaygisiz et al., 2016). A Classificação de Angle também possui limitações. Foi questionada por ser considerada um método qualitativo das oclusopatias, ou seja, baseado somente no posicionamento anteroposterior dos dentes, não quantificando o desvio e não citando as alterações verticais ou transversais (Garbin et al., 2010). No decorrer do tempo e para suprir as deficiências do método, estudos (Brin et al.,2000; Gudipaneni et al., 2018) foram realizados acrescentando novos critérios à classificação original, como: mordida cruzada anterior e posterior, mordida aberta anterior e posterior, apinhamento superior e inferior, diastemas, mal posicionamento dentário individual, overjet e overbite. Tais variáveis também foram utilizadas neste estudo complementando a Classificação de Angle. 
Ressalta-se o desfecho observado quanto à alta necessidade de tratamento ortodôntico nos jovens de 12 anos de idade, o que torna-se primordial, sob a perspectiva da saúde pública, a formulação de ações e programas com ênfase na educação em saúde bucal e prevenção das oclusopatias, direcionados à população, para a promoção e conscientização da manutenção da saúde bucal e do estímulo do contato precoce à assistência odontológica, evitando e/ou reduzindo as sequelas dos principais problemas em oclusão. Além disso, sugere-se que a possibilidade de monitoramento regular da demanda com necessidade de tratamento ortodôntico, em um sistema público de saúde, promoveria o melhor controle do número de indivíduos encaminhados, com atendimentos iniciados, em andamento e concluídos. Com esta gestão, proporcionaria uma melhor avaliação da necessidade de ampliação do acesso aos serviços odontológicos, visando os cuidados ortodônticos. Já, sob uma visão epidemiológica, o monitoramento periódico da necessidade de tratamento ortodôntico permitiria a comparação desta condição em diferentes períodos e a verificação da eficácia das ações e estratégias implementadas.

\section{Conclusão}

A prevalência das oclusopatias e da necessidade normativa de tratamento ortodôntico foi elevada, sendo que aproximadamente $1 / 5 \mathrm{com}$ necessidade de tratamento obrigatório. Os índices foram eficazes para a identificação das oclusopatias, entretanto por avaliarem aspectos clínicos distintos, poderiam ser utilizados concomitantemente para aprimorar o diagnóstico das oclusopatias.

\section{Agradecimentos}

O presente trabalho foi realizado com apoio da Coordenação de Aperfeiçoamento de Pessoal de Nível Superior Brasil (CAPES) - Código de Financiamento 001

\section{Referências}

Alhammadi, M. S., Halboub, E., Fayed, M. S., Labib, A. \& El-Saaidi C. (2018). Global distribution of malocclusion traits: a systematic review. Dental Press Journal of Orthodontics, (23)6, 23-40.

Almeida, A. B. \& Leite, I. C. (2013). Orthodontic treatment need for Brazilian schoolchildren: a study using the Dental Aesthetic Index. Dental Press Journal of Orthodontics, (18)1, 103-109.

Angle, E. H. (1899). Classification of malocclusion. Dental Cosmos, 41, 248-264, 350-357.

Ashari, A. \& Mohamed, A. M. (2016). Relationship of the Dental Aesthetic Index to the oral health-related quality of life. Angle Orthodontist, 86(2), 337-342.

Asiry, M. A. \& AlShahrani, I. (2019). Prevalence of malocclusion among school children of Southern Saudi Arabia. Journal of Orthodontic Science, (20), 8-2.

Baram, D., Yang, Y., Ren, C., Wang, Z., Wong, R. W. K. \& Hägg, U., et al. (2019). Orthodontic treatment need and the psychosocial impact of malocclusion in 12-year-old Hong Kong children. Scientific World Journal, 2685437.

Borzabadi-Farahani, A. An insight into four orthodontic treatment need indices. (2011). Progress in Orthodontics, 12(2), 132-42.

Brasil. Ministério da Saúde. Secretaria de Atenção à Saúde. Secretaria de Vigilância Sanitária. SB Brasil 2002-2003: Pesquisa Nacional de Saúde Bucal: principais resultados. Brasília: Ministério da Saúde, 2003

Brasil. Ministério da Saúde. Secretaria de Atenção à Saúde. Secretaria de Vigilância Sanitária. SB Brasil 2010: Pesquisa Nacional de Saúde Bucal: manual da equipe de campo. Brasília: Ministério da Saúde, 2009.

Brasil. Ministério da Saúde. Secretaria de Atenção à Saúde. Secretaria de Vigilância Sanitária. SB Brasil 2010: Pesquisa Nacional de Saúde Bucal: principais resultados. Brasília: Ministério da Saúde, 2012.

Brin, I., Weinberger, T. \& Ben-Chorin, E. Classification of occlusion reconsidered. (2000). European Journal of Orthodontics, 22(2), 169-174.

Chaves, M. M. (1986). Odontologia social. (3a ed.) Artes Médicas.

Doğramac1, E. J. \& Rossi-Fedele, G. (2016). Establishing the association between nonnutritive sucking behavior and malocclusions: a systematic review and meta-analysis. The Journal of the American Dental Association, 147(12), 926-934.e6. 
Esa, R., Razak, I. A. \& Allister, J. H. (2001). Epidemiology of malocclusion and orthodontic treatment need of 12-13-year-old Malaysian schoolchildren. Community Dental Health Journal, 18(1):31-36.

Garbin, A. J. I., Perin, P. C. P., Garbin, C. A. S. \& Lolli, L. F. (2010). Malocclusion prevalence and comparison between the Angle classification and the Dental Aesthetic Index in scholars in the interior of São Paulo state - Brazil. Dental Press Journal of Orthodontics, 15(4), 94-102.

Gudipaneni, R. K., Aldahmeshi, R. F., Patil, S. R. \& Alam, M. K. (2018). The prevalence of malocclusion and the need for orthodontic treatment among adolescents in the northern border region of Saudi Arabia: an epidemiological study. BMC Oral Health, 18(1), 16.

Hassan, R. A. \& Rahimah, A.K. (2007). Occlusion, malocclusion and method of measurements - an overview. Archives of Orofacial Sciences, 2, 3-9.

Herkrath. A. P. C. Q., Vettore, M. V., Queiroz, A. C., Alves, P. L. N., Leite, S. D. C. \& Pereira, J.V., et al. (2019). Orthodontic treatment need, self-esteem, and oral health-related quality of life among 12-yr-old schoolchildren. European Journal of Oral Sciences, 127(3), 254-260.

Kaygisiz, E., Uzuner, F. D. \& Taner L. A. (2016). Comparison of three orthodontic treatment indices with regard to angle classification. Journal of Clinical Pediatric Dentistry,40(2), 169-174.

Lin, M., Xie, C., Yang, H., Wu, C. \& Ren, A. (2020). Prevalence of malocclusion in chinese schoolchildren from 1991 to 2018: A systematic review and metaanalysis. International Journal of Paediatric Dentistry, 30(2), 144-155.

Lombardo, G., Vena, F., Negri, P., Pagano, S., Barilotti, C. \& Paglia, L., et al. (2020). Worldwide prevalence of malocclusion in the different stages of dentition: a systematic review and meta-analysis. European Journal of Paediatric Dentistry,21(2), 115-122.

Moyers, R.E. (1988). Handbook of orthodontics. Chicago: Year Book Medical.

Nayak, P. P., Prasad, K. \& Bhat, Y. M. (2015). Orthodontic treatment need among special health care needs school children in Dharwad, India: a comparative study. Journal of Orthodontic Science, 4(2), 47-51.

Nunes-Neto, T. A., Thomaz, E. B., Ferreira, M. C., Santos, A. M. \& Queiroz, R.C. (2014). Problemas de espaço dentário em adolescentes brasileiros e fatores associados. Ciência \& Saúde Coletiva, 19(11), 4555-4568.

Pinto, E. M., Gondim, P. P. C. \& Lima, N. S. L. (2008). Critical analyses of some malocclusions register and evaluation methods. Revista Dental Press de Ortodontia e Ortopedia Facial,13(1), 82-91.

Proffit, W. R., Fields, H. W., Sarver, D. M. \& editors. (2013). Contemporary orthodontics. (5a ed.), Elsevier.

Savara, B. S. \& Steen, J. C. (1978). Timing and sequence of eruption of permanent teeth in a longitudinal sample of children from Oregon. The Journal of the American Dental Association, 97(2), 209-214.

Silva, L. F. G., Thomaz, E. B. A. F., Freitas, H. V., Ribeiro, C. C. C., Pereira, A. L. P. \& Alves, C. M. C. (2016). Self-perceived need for dental treatment and related factors: a cross-sectional population-based study. Brazilian Oral Research,30 (1), e55.

Ting, T. Y., Wong, R. W. \& Rabie, A.B. (2011). Analysis of genetic polymorphisms in skeletal Class I crowding. American Journal of Orthodontics and Dentofacial Orthopedics, 140 (1), e9-15.

Thomaz, E. B., Cangussu, M. C. \& Assis, A.M. (2013). Malocclusion and deleterious oral habits among adolescents in a developing area in northeastern Brazil. Brazilian Oral Research, 27(1), 62-69.

Todor, B. I., Scrobota, I., Todor, L., Lucan, A. I. \& Vaida, L.L. (2019). Environmental factors associated with malocclusion in children population from mining areas, western romania. International Journal of Environmental Research and Public Health,16(18), 3383.

Tolessa, M., Singel, A. T. \& Merga, H. (2020). Epidemiology of orthodontic treatment need in southwestern Ethiopian children: a cross sectional study using the index of orthodontic treatment need. BMC Oral Health, 20(1),210.

van Wyk, P. J. \& Drummond, R. J. (2005). Orthodontic status and treatment need of 12-year-old children in South Africa using the Dental Aesthetic Index. Journal of the South African Dental Association, 60(8), 334-336, 338.

Vedovello, S. A. S., Santos, P. R., Carvalho, A. L. M., Vedovello Filho, M., Ambrosano, G. M. B., Pereira, A. C., et al. (2019). Exploring the perception of orthodontic treatment need using the Dental Aesthetic index and index of Orthodontic Treatment Need. American Journal of Orthodontics and Dentofacial Orthopedics, 156(6), 818-822.

World Health Organization. (1987). Oral health surveys: basic methods. (3a ed.), Geneva: ORH/EPID.

World Health Organization. (2003). The world oral heath report 2003. Geneva: WHO. 